\title{
Stefan Zweig face à Cicéron, ou la biographie comme miroir de soi
}

\author{
Michel Magniez \\ Université de Strasbourg II
}

Le recueil intitulé Die Sternstunden der Menschheit occupe une place toute particulière dans la foisonnante production littéraire de Stefan Zweig, composée pour l'essentiel de nouvelles (Vingtquatre heures de la vie d'une femme, La confusion des sentiments) et de biographies consacrées à des personnages illustres (Marie Stuart, Erasme, Magellan, Marie-Antoinette, Balzac, Montaigne...). Cette œuvre, dont une partie a été traduite en français sous le titre LesHeures glorieuses de l'Humanité, rassemble dans sa version allemande définitive quatorze textes, dont chacun traite l'une des heures fatales qui ont marqué à jamais l'histoire du monde. Cicero est l'une de ces quatorze «miniatures historiques", selon les termes-mêmes de Stefan Zweig, dans lesquelles l'auteur cherche à saisir «les instants sublimes» et tragiques, qui, « de 
manière irrévocable», ont déterminé «le sort de toute l'humanité » (p. 8). Cicero partage avec les treize autres textes du recueil une manifeste indétermination générique, car chacune de ces "miniatures historiques», d'une longueur approximative de vingt-cinq pages, se situe au croisement de la nouvelle et de la biographie.

$\mathrm{Au}$ cours de cette œuvre, Stefan Zweig investit ses propres interrogations, ses doutes et ses angoisses dans la représentation qu'il donne de Cicéron. Relatant la vie de l'orateur romain, il trace en fait son propre portrait, et fait de Cicéron à la fois le premier représentant des valeurs humaines auxquelles il croit, mais également un double de lui-même, dont les idéaux humanistes ont été vaincus par la violence et la force brutale d'un régime dictatorial.

Il faut d'abord évoquer l'intrigue et les grands traits de l'action de cette nouvelle, toujours inédite en français, et parue uniquement en allemand et en anglais. Plusieurs indices laissés par Stefan Zweig dans son texte permettront de montrer comment s'établit l'analogie entre le philosophe romain de l'Antiquité et l'auteur autrichien du $20^{\mathrm{e}}$ siècle. Cicéron devient alors, sous la plume de Stefan Zweig, un exemplum particulièrement noble pour représenter les valeurs universelles que se doit de défendre tout penseur humaniste, même au cours des périodes les plus sombres de l'Histoire.

\section{Trame narrative et réalité historique}

L'action de cette "heure glorieuse », divisée en huit chapitres, se déroule entre les années 49 et 43 av. J-C. 
Jules César, qui s'est rendu maître de Rome, a poussé l'orateur Cicéron à quitter la vie politique. Cicéron s'adonne alors à la philosophie et à la vie contemplative, jusqu'au jour où César est assassiné. Le penseur tente alors de saisir l'occasion pour rétablir la République romaine, mais il renonce à ce projet face à l'apathie et à la corruption des élites de la nation, qui s'ajoute à la passivité coupable du peuple romain. Déçu, il se retire donc une deuxième fois du monde politique, pour rédiger cette fois son traité Des devoirs - le De Officiis (chapitres I à IV). Cependant, il retourne peu de temps après à Rome car l'ancien consul Antoine, à la tête d'une armée de mercenaires, veut s'emparer du pouvoir. Pour lutter contre cet homme cupide et immoral, Cicéron prononce au Sénat ses quatorze Philippiques, dans lesquelles il attaque violemment Antoine (chapitre V). Ce dernier forme alors une alliance avec ses adversaires, Octave et Lépide, et demande la mort de Cicéron. Le philosophe, qui en est averti, renonce pourtant à fuir et meurt assassiné. Antoine fait alors accrocher la tête et les mains de l'orateur au-dessus des Rostres, les tribunes du Sénat, en guise d'avertissement au peuple romain (chapitres VI à VIII).

Le synopsis de la miniature historique, ainsi que le détail du texte, révèle une grande part de fidélité, dans le récit de Stefan Zweig, aux sources antiques et historiques. Le schéma de son récit s'inspire surtout des Vies parallèles de Plutarque, en particulier La Vie de Cicéron et La Vie d'Antoine, dont il reprend les principales données chronologiques et nombre de précisions biographiques. Pour relater le passage précis de la mort de Cicéron, Zweig s'est en outre référé au fragment de l'Histoire de Rome de Tite-Live (Ab Vrbe Condita, CXX), qui nous a été transmis par Sénèque le Rhéteur (Suasoriae, VI, 17). TiteLive propose en effet une description très précise des derniers 
instants vécus par l'orateur, et Zweig le suit de près en indiquant notamment les dernières paroles que Cicéron adressa à ses esclaves ("Laisse-moi mourir dans ce pays que j'ai sauvé »), et en évoquant le courage de l'orateur face à la mort et le dévouement de ses esclaves.

Stefan Zweig s'en tient donc apparemment le plus fidèlement possible à la vérité historique, mais ce serait méconnaître le sens profond de l'œuvre que de la considérer comme un simple compte-rendu objectif de la vie de Cicéron.

\section{Cicéron comme portrait de Stefan Zweig}

Il faut d'abord rappeler les circonstances de la création de l'œuvre. D'après les renseignements fournis par le journal intime et la correspondance de Stefan Zweig, on peut établir que l'écriture de Cicero a eu lieu entre le 15 septembre et le 11 octobre 1939. Zweig, qui a fui la dictature nazie et l'Annexion de l'Autriche, est alors en Angleterre, près de Bath et il n'a pas encore quitté Londres pour le Brésil. Sur le plan international, la guerre est déclarée depuis le début du mois de septembre 1939, à la suite de l'invasion de la Pologne par l'Allemagne.

On notera en premier lieu la similitude de la situation personnelle des deux écrivains : Stefan Zweig insiste sur le fait que la plupart des événements qu'il relate se déroulent alors que Cicéron a soixante ans. Né en 1881, Stefan Zweig lui-même entame sa cinquante-huitième année lorsqu'il écrit Cicéro. Serge Niémetz, dans sa grande biographie de Stefan Zweig intitulée Stefan Zweig, le voyageur et ses mondes, indique à quel 
point l'auteur autrichien était profondément angoissé par l'approche du « jour fatal » de sa soixantième année.

Autre détail biographique sur lequel Zweig s'appesantit : Cicéron vient de se remarier avec une femme plus jeune que lui, Tullia : «Et puis, dernière sagesse, ce sexagénaire commet la plus douce des folies de l'âge, il prend une femme jeune [...] pour jouir en artiste de la beauté de la vie, non dans le marbre ou dans les vers, mais sous sa forme la plus sensuelle et la plus séduisante. » (p. 254) Zweig lui-même, deux ans auparavant, a quitté sa femme, Friderieke, pour épouser en 1938 la jeune Charlotte Altmann, qu'il a rencontrée en 1934 alors qu'elle avait 26 ans et que lui en avait 53 .

D'autre part, le récit de Stefan Zweig évoque les nombreuses périodes d'exil que connut Cicéron : «Il a dû fuir en exil, du jour au lendemain, condamné par le sénat et abandonné par le peuple». "Pour la seconde fois, Marcus Tullius Cicéron a quitté le monde pour fuir dans la solitude » (p. 251-252). Cette situation rappelle les circonstances dans lesquelles Zweig a écrit son œuvre. Exilé à Bath, en Angleterre, il écrit dans ses Journaux le 3 septembre 1939: "Maintenant commence pour moi une autre vie, j'ai perdu ma liberté et mon indépendance » $(1995$, p. 396). Son autobiographie, Le Monde d'hier, Souvenirs d'un Européen, montre à quel point le statut d'exilé puis d'apatride a marqué Zweig, toujours en fuite vers de nouvelles terres : "Car retranché de toutes racines, et même de la terre qui avait nourri ces racines, je l'ai été comme peu d'hommes, véritablement, le furent jamais » (2006, p. 8)

Cette fuite perpétuelle, Zweig la retrouve et la reconnaît en Cicéron : «Dans les derniers mois, Cicéron se cache tantôt dans l'une, tantôt dans l'autre de ses propriétés à la campagne, 
repartant toujours dès qu'un danger le menace, mais ne lui échappant jamais totalement.» Zweig mêle au récit biographique la constatation personnelle que lui fournit sa propre expérience : «Et en effet, par deux fois, par trois fois, ce réprouvé semble décidé à prendre la fuite. Il prépare tout, il informe ses amis, il embarque, il se met en route. Mais Cicéron s'arrête toujours au dernier moment; qui a un jour connu la tristesse de l'exil ressent même dans le danger la volupté de la terre familière et l'indignité d'une vie passée à fuir » (p. 271). Le récit biographique est donc enrichi, sous couvert de remarques générales, par des allusions à la vie personnelle de l'auteur.

Cette union entre biographie et autobiographie apparaît également dès la première phrase du récit, dans l'attitude de retrait, loin de la scène publique, adoptée par Cicéron après la victoire de César : « Le plus sage qu'un homme intelligent et pas très courageux puisse faire lorsqu'il en rencontre un plus fort que lui, c'est de l'éviter et d'attendre sans honte le moment où la voie lui redevient libre » (p. 250). Cette affirmation témoigne en fait d'une attitude constante de la part de Stefan Zweig, qui écrivait dans Le monde d'hier au sujet de la première guerre mondiale : «Il ne restait dès lors qu'une seule chose à faire : se replier sur soi-même et se taire aussi longtemps que dureraient la fièvre et le délire des autres » (2006, p. 281). La première phrase du récit montre donc que cette "miniature historique » est au croisement de la biographie et de l'autobiographie.

Stefan Zweig, à cette époque, est d'ailleurs lui-même contraint au retrait et à la résignation. Il écrit dans son autobiographie, au sujet de l'automne 1939: «Au cours de ces mois, j'avais quitté Londres et m'étais retiré à la campagne à Bath. Jamais dans ma vie je n'avais éprouvé plus cruellement 
l'impuissance de l'homme face aux événements mondiaux. » (2006, p. 499) De même Cicéron «tente de s'enseigner à luimême qu'un vrai sage doit apprendre la résignation, la véritable dignité de son âge et de ses années » (2000, p. 255). À l'heure où Zweig tente de se jeter à corps perdu dans le travail, entreprend son autobiographie et tente de parachever son œuvre, il écrit de Cicéron: "il ne peut rien arriver de plus heureux à un homme d'esprit que la mise à l'écart de la vie publique et politique ; le penseur, l'artiste est poussé hors d'une sphère indigne de lui [...] et il est ramené à sa sphère intérieure, intangible et indestructible. Toute forme d'exil donne à l'homme d'esprit l'impulsion qui le conduit vers le recueillement intérieur, et ce malheur béni frappe Cicéron au moment le plus propice.»(p. 251) Tout indique que Zweig décrit ainsi sa propre volonté et sa situation personnelle par le biais de Cicéron.

Cicéron, dans le texte de Zweig, est d'ailleurs déçu par la politique, "la sphère publique », et offre une vision noire du peuple et des hommes d'action, qui ne cherchent qu'à assouvir leur désir de gloire et de pouvoir. Là encore, c'est une constante de la pensée philosophique de Stefan Zweig qui apparaît à travers cette biographie. Zweig écrivait en effet à Romain Rolland, un mois avant de commencer la rédaction de Cicero : «Vous savez que j'ai refusé depuis longtemps ma confiance non seulement à la politique, mais surtout aux politiciens - je l'ai témoigné par monFouché et par mon Erasme. » (Lettre datée du 23 août 1939). Cicéron reprend donc ce leitmotiv de la condamnation de la res publica, la sphère publique et politique, mais il y ajoute, comme par compensation, l'éloge de la «vie intérieure», de la "contemplation», et de la réflexion philosophique sur soi et sur le monde. 
À l'inverse, le peuple lui-même et les aléas de la vie publique sont sources de déception pour Cicéron comme pour Zweig : lui qui a été l'un des écrivains les plus admirés dans toute l'Europe est chassé de sa patrie et voit ses livres brûlés dans son propre pays. De même, Cicéron a été "porté en triomphe», "couronné par le peuple», et «honoré par le Sénat», puis « condamné par ce même sénat et abandonné par ce même peuple. » Tous deux ont été, du jour au lendemain, " au plus haut puis au plus bas de la faveur populaire », "fort admirés et fort haïs », d'abord adulés et célébrés, puis méprisés et rejetés (p. 251-252).

Toutes ces réflexions amènent forcément à réinterpréter le taedium vitae, à savoir le "dégoût de la vie ", la fatigue, la lassitude, ressentis par Cicéron dans le récit de Stefan Zweig: «La fatigue, le scepticisme envahissent de plus en plus Cicéron. »; "Cicéron est envahi à la fois par la fatigue et la sagesse, deux sentiments qui se ressemblent souvent bien dangereusement. »; "Une fatigue non seulement physique et nerveuse, mais une fatigue de la vie, une nostalgie mystérieuse de son terme et de sa terre, s'est emparée de lui » (p. 272). On note, en écho à ces remarques, dans les Journaux de Stefan Zweig, en septembre 1939: «Triste. Las. Je n'ai qu'un seul espoir : que cette guerre se termine bientôt. [...] Ma situation ici est odieuse - isolé, sans pouvoir ni occasion de m'exprimer [...] » (1995, p. 406). Le mal-être de l'écrivain est patent: « Je n'ai plus le choix, je dois rester où je suis; ma vie de toute façon ne vaut plus grand chose» (1995, p. 407). Son malaise s'accroît jour après jour, et son journal est émaillé de constats d'impuissance et d'attentes déçues : «Rien de nouveau », «Rien d'important», «Je suis las de penser à l'avenir», «Journée de dépression», «Cette époque me 
fatigue », "Cette guerre m'inspire un sentiment de malaise » (1995, p. 393-410, passim). Comment, enfin ne pas mettre en relation l'attitude de Cicéron, qui appelle la mort de ses vœux, et se laisse tuer pour échapper aux tourments et aux troubles de son époque, avec le suicide commis par Stefan Zweig deux ans plus tard en compagnie de son épouse? À Cicéron qui déclare : «Je suis fatigué de fuir, et fatigué de vivre », font écho les dernières lettres de Zweig à ses proches avant son suicide : «J'étais trop fatigué pour supporter cela», «j'étais oppressé par notre vie de nomades », " mes forces sont épuisées par des années d'errance sans patrie » (Niémetz, 1996, p. 554-555).

\section{Cicéron comme incarnation des valeurs humanistes}

Il est difficile de ne pas reconnaître, dans la description que fait Stefan Zweig de la Guerre civile à Rome, une allusion à peine voilée aux événements historiques qui ébranlent le monde en cette année 1939. Rappelons que dans son autobiographie,Le monde d'hier, Stefan Zweig associait déjà la Guerre civile romaine à la Seconde Guerre mondiale : il commence ainsi le chapitre "L'agonie de la paix», consacré aux années 19341940, par une citation de la tragédie de Shakespeare intitulée Jules César: "Le soleil s'est couché sur Rome. Pour nous, le jour touche à sa fin. Les nuages, les dangers, les brumes se rapprochent ; c'en est fini de notre action » (2006, p. 454). Ces paroles prononcées par Titinius, l'un des conjurés, après la mort de Brutus et Cassius, symbolisent pour Stefan Zweig la montée en puissance du nazisme jusqu'à l'éclatement de la guerre en 1939. De même, dans Cicero, les légions et cohortes romaines sont la transposition antique des armées allemandes, 
et Antoine, dictateur cupide, immoral et sans scrupule, est sans conteste l'une des incarnations de la « violence pure » au même titre qu'Hitler.

Il est caractéristique, à cet égard, que Stefan Zweig parle constamment de «l'Empire romain » pour désigner un État qui à l'époque de Cicéron, est encore une République. Le choix de l'expression «das römische Reich » n'est pas anodin, et renvoie directement au «deutsches Reich » dont Hitler veut étendre le pouvoir et la domination.

Cicéron devient alors le représentant des valeurs humanistes en lesquelles croit Stefan Zweig. Il est, pour celui-ci, le modèle de l'homme d'esprit qui a su, malgré l'adversité, sauvegarder sa liberté intérieure. Il écrivait à Romain Rolland le 11 octobre 1939, alors qu'il achevait d'écrire Cicéron : "J'ai été surpris en lisant son De re publica et son De Officiis. Il est notre homme, lui qui mourut pour nos idées, en des temps qui ressemblaient si cruellement aux nôtres. » De fait, Zweig rappelle la lutte de Cicéron, dans le De Officiis, contre l'impérialisme et les guerres de conquêtes: "D’un regard de prophète, il prévoit le déclin de Rome provoqué par la succession trop rapide de ses victoires et de ses conquêtes mondiales, malsaines car uniquement militaires. [...] Et à chaque fois qu'un peuple prend par les armes leur liberté à d'autres peuples, il perd par une mystérieuse vengeance l'admirable force qui était sienne dans son isolement » (p. 263). Ces phrases ne peuvent résonner que comme un écho aux événements de septembre 1939, et comme un avertissement aux peuples responsables de la guerre. «Tandis que les légions font marche sous le commandement de généraux ambitieux, [...] une voix s'élève contre ce dangereux triomphe : en effet, 
Cicéron a vu comment la semaison sanglante des guerres de conquête faisait croître la récolte plus sanglante encore des guerres civiles » (p. 263).

"Cet impuissant défenseur de l'humanité », devenu le porte-parole de Stefan Zweig, se fait l'adversaire de la dictature et de la dominatio unius (c'est-à-dire «la domination d'un seul »), prônant en lieu et place l'adjumenta hominum, "l'aide réciproque entre les hommes ». "Pour lui, le despotisme viole toute forme de droit. La véritable harmonie ne peut naître dans une communauté que lorsque l'individu, au lieu de tirer un profit personnel de sa position publique, fait passer ses intérêts privés après ceux de la collectivité. » (p. 262)

Zweig s'interroge cependant avant tout dans Cicero sur le pouvoir de l'hommed'esprit face à la barbarie et à la violence. Il montre, par le biais du philosophe romain, la tragique défaite de l'humanisme face à la brutalité, et sa propre défaite personnelle. Cicéron est d'abord vaincu par César car «Comme toujours, les cohortes se sont révélées plus fortes que les mots » (p. 250). "Le plus grand défenseur de la république romaine» est enfin assassiné par de " petits bandits » illettrés, à la solde d'Antoine, désigné comme le dictateur, «le grand bandit». Zweig appelait d'ailleurs les Nazis « les canailles brunes ». La fin de Cicéron est son dernier appel, dérisoire, à la lutte « contre la brutalité, contre la rage du pouvoir et contre l'anarchie» (p. 275)

Ainsi Stefan Zweig, par «ce symbole tragique de la république crucifiée ", fait de la biographie une arme contre "l'éternelle injustice de la violence », à laquelle succombèrent la république romaine au premier siècle av. J.-C. et le monde entier en 1939. 


\section{D’une biographie à l'autre : Érasme et Cicéron}

Il convient pour conclure de mettre en valeur le rapprochement qui doit être opéré entre Cicéron et Erasme, héros tous deux de biographies de Stefan Zweig. Ne parvenant pas à se mettre au travail, Stefan Zweig note dans son journal intime, le 10 septembre 1939: «Je pense que l'autobiographie ne serait pas indiquée. Quant à écrire des romans, je n'en ai pas le courage. Peut-être le mieux serait il un ouvrage comme mon Erasme. » Et il complète cette réflexion le 15 septembre : « Je vais commencer mon Cicéron, j'ai déjà perdu trop de temps avec cette stupidité que sont la politique et la guerre. » (1995, p. 402 et 404)

De fait, l'analogie entre Erasme et Cicéron est étonnante, et elle montre que l'affirmation d'un lien fort entre l'auteur et le sujet de la biographie est une constante des biographies de Zweig. Analysant la biographie d'Erasme, François Dosse écrit dans Le Pari biographique. Ecrire une vie: «Dans cette attirance pour ces personnages qui refusent toute allégeance au nom de la liberté, on saisit là encore le biographe sous les traits du biographé : "Erasme ne veut se lier à personne... En son for intérieur, il n'a jamais reconnu l'autorité d'un supérieur." » (1980, p. 80)

L'étude de l'éthos biographique dans Cicéron permet donc de compléter cette analyse et d'ébaucher une analyse plus complète de l'œuvre de Zweig: le biographe se projette luimême dans le sujet de sa biographie, et y projette également les angoisses liées à son époque et les doutes liés à sa condition. Cicéron, comme Erasme, est la preuve qu'il faut, selon Zweig, « écrire en symboles » pour saisir la complexité de l'Histoire, ancienne ou contemporaine, et pour faire entendre sa voix malgré le tumulte du monde et des hommes. 


\section{Bibliographie}

Dosse, François. 2005, Le Pari biographique. Écrire une vie, Paris, La Découverte.

NiÉmETZ, Serge. 1996, Stefan Zweig. Le voyageur et ses mondes, Paris, Belfond.

Plutarque, 1975-1978, Vies Parallèles, tomes IX, XII, XIII, XIV, Paris, Les Belles Lettres, coll. « C.U.F.»

SÉNÈQUE, 1992, Sentences, divisions et couleurs, des orateurs et des rhéteurs :controverses et suasoires, Paris, Aubier.

ZWEIG, Stefan. 1935, Erasme, Paris, Grasset.

—, 1991-1996, CEuvres complètes, Paris, Lgf, coll. « La Pochothèque ».

—, 1995 [1986], Journaux, Paris, Lgf.

-, 2000 [1927], Sternstunden der Menschheit, Vierzehn historische Miniaturen, Francfort-sur-le-Main, Fischer Taschenbuch Verlag.

—, 2004 [1996], Les très riches heures de l'humanité, Paris, Belfond.

—, 2006 [1982], Le monde d'hier, Souvenirs d'un Européen, Paris, Albin Michel. 


\title{
Résumé
}

S'inspirant des œuvres antiques qui sont à sa disposition, Stefan Zweig rédige en septembre 1939 une courte biographie de Cicéron, dans laquelle il relate en particulier les dernières années de la vie du philosophe. Le biographe retrace alors des événements lointains qui ont marqué l'histoire de l'Europe, en lesquels il trouve plus d'une similitude avec les soubresauts dont sa propre époque est agitée. Parler de Cicéron devient alors pour Stefan Zweig un moyen de représenter sa condition d'exilé, mais aussi de réfléchir sur le déclin de son époque et sur l'apparente défaite des valeurs humanistes qui l'animent depuis toujours.

\begin{abstract}
Inspired by the ancient books of Plutarch and Titus Livius, Stefan Zweig writes in September 1939 a short biography of Marcus Tullius Cicero. He tells us how Cicero lived the last years of his life, and he relates how the Roman Republic came to an end with Cicero's death. However, the events that occur during those years are very similar to those that Zweig himself is witnessing in 1939. Writing about Cicero thus becomes for Stefan Zweig a way of depicting his own situation of exile as well as an opportunity to meditate on the decline of his own era with the beginning of the Second World War, and on the apparent collapse of the moral values in which he sincerely believes.
\end{abstract}

